\title{
Uniform Circular Broadband Beamformer with Selective Frequency Invariant Region
}

\author{
Xin Zhang ${ }^{*}$, Wee Ser ${ }^{*}$, Zhang Zhang ${ }^{*}$ and Anoop Kumar Krishna ${ }^{\dagger}$ \\ * Center of Signal Processing \\ School of Electrical and Electronic Engineering \\ Nanyang Technological University, 50 Nanyang Avenue, Singapore 639798 \\ Tel: +65 67905912, Fax: +65 67912383 \\ E-mail: zhang_xin@pmail.ntu.edu.sg, ewser@ntu.edu.sg, zhan0090@ntu.edu.sg \\ ${ }^{\dagger}$ Research \& Development Department, ST Microelectronic \\ 5A, Serangoon North Avenue 5, Singapore 554574
}

\begin{abstract}
Frequency-Invariant (FI) beamformer is a type of beamformer that has flat spatial response over a wide range of frequency. In most of the algorithms presented today, this FI characteristic is true for all angles including both main lobe and side lobe. In this paper, a novel uniform circular broadband beamformer with frequency-invariant characteristic is proposed. It attempts to optimize the FI beampattern solely for the main lobe where the signal of interest is from and relax the FI requirement on the side lobe. As a result, the beamformer achieves better FI response for the desired region and it is completed in one single step. In order to achieve this goal, a new objective function with a quadratic constraint is designed. In addition, the constraint function allows the FI character to be accurately controlled over the specified bandwidth at the expense of other parts of the spectrum which are not of concern to the designer. State-of-the-art optimization method such as Second Order Cone Programming (SOCP) is used to solve this complex optimization problem with high efficiency and accuracy. Simulation result shows that the proposed beamformer can obtain better performance in achieving FI for the main lobe.
\end{abstract}

\section{INTRODUCTION}

Systems that employ microphone arrays are very often involved in processing broadband signals, such as speech signal. In the literature presented today, there are several ways of designing a broadband beamformer [3] [5] [6]. One approach is to use narrowband decomposition [2]. In this technique, the signal received at each sensor is transformed into the frequency domain, and each frequency band is then treated as an independent narrowband beamformer. This approach is computational intensive. Alternatively, adaptive broadband beamformer is used for broadband signal [4]. It employs tapped-delay lines or linear transversal filters with adaptive coefficients to generate appropriate beampattern so as to suppress undesirable interference, one example is Frost beamformer. An adaptive array with $\mathrm{K}$ sensors can produce K-1 constraints on the beam pattern of the array at a single frequency. If each sensors have L-tap FIR filter, then the same constraints can be applied at $\mathrm{L}$ different frequencies. In order to have identical beampattern over a continuous range of frequencies, for adaptive beamformer, large number of sensors and taps are required. Hence high computational complexity is expected. The third approach of designing broadband beamformer is to design a beamformer with frequency-invariant beampattern (FIB). In FIB, the array has constant spatial response over a wide range of frequency bands. One advantage of them is that they are able to attenuate broadband interference with fewer number of adaptive filter coefficients, hence resulting in lower computational complexity. Among the existing available techniques, they can be classified into three categories. One is the FIB optimization based on FIR or IIR coefficients [7]-[10]. This technique employs some analytical relations between frequency responses of filters located on different sensor array elements of the FIB, with utilization of a differentiation filter at the beamformer output and with or without utilization of multi-rate techniques. A limitation pertaining to this group is that it restricts to only linear array configuration. Being linear array, the resolution at broadsight is better than that at its endfire position. The second category is beampattern optimization for single frequency [13]. In this approach, some desired beamformer response over angle and frequency is first defined, then the vector of the beamformer coefficients are optimized in order to minimize the error between the desired and real pattern. One weakness for this approach is the choice of the desired pattern has to be decided skillfully. The third type is FIB optimization based on array configuration. This type of beamformer makes use of array geometry specialty to remove the frequency dependency of the received signal [14]. One example is the uniform circular array with frequencyinvariant characteristic proposed recently. Being a circular array, it receives signal from all direction with equal resolution. Hence it resolves the problem created by linear array. In paper [1], by exploiting the geometric advantage of the circular array, a frequency compensation network is designed to remove the frequency dependency of the received signal and the outcome are summed to produce a FI beampattern. To do so, the array snapshot is first transformed to phase modes via an Inverse Discrete Fourier Transform (IDFT), followed by applying spatial weights. The spatial 
weights, which govern the far field pattern of the array, can be designed by conventional 1-D digital filter design techniques such as Parks-McClellan Algorithm. In this method, the FI beampattern is optimized for the entire band including main lobe and side lobes in two separate steps. Ideally, the compensation filter and the beam weight which determine the array beampattern can be optimized jointly. However, using the method presented in [1], large number of variables and constraints for this direct approach make it difficult to solve.

As a result, in this paper, a novel algorithm with new objective function and a quadratic constraint is proposed. It aims to achieve an FI beampattern for the main lobe where the signal of interest is from and relax the FI requirement at the side lobes. In doing so, it is able to achieve FI characteristic in one single step optimization. Furthermore, the constraint function accurately controls the FI character over a specified bandwidth which may be an interest to the designer. By focusing the FI characteristic along the desired direction only, and relaxing the FI requirement at other direction, the free degrees of freedom make it possible to design an FIB array in single step procedure. This novel beamformer may find its application in vehicular environment, where the desired signal and the noise are both broadband. In such case, the spectrum of noise is much broader than that of speech, the proposed beamformer focuses on the spectrum of the desired speech signal at the desired direction, while attenuate the broadband noise coming from other direction and the undesired portion of the spectrum.

The remaining paper is organized in the following way: In section II, problem formulation is discussed. In section III, the design of the beamforming weight using SOCP is presented. Numerical results are given in section IV and finally, conclusions are drawn in section $\mathrm{V}$.

\section{PROBLEM FORMULATION}

In Fig 1, a layer of $\mathrm{K}$ microphones are distributed uniformly in a circle. Each omni-directional sensor is located at $\left\{r \cos \phi_{k}, r \sin \phi_{k}\right\}$ where $r$ is the radius of the circle, $\phi_{k}=2 \pi k / K$ and $k=0, \ldots, K-1$. In this configuration, the inter-sensor spacing is fixed at $\lambda / 2$, where $\lambda$ is the smallest wavelength of the array to be operated and is denoted as $\lambda_{\text {min }}$. Half wavelength is chosen because it is widely used in linear array to avoid spatial aliasing. Hence the radius is given by [1]:

$$
r=\lambda_{\min } / 4 \sin (\pi / K)
$$

Assuming the circular array is on a horizontal plane, the steering vector is

$$
\mathbf{a}(f, \phi)=\left[e^{\frac{j 2 \pi f r \cos \left(\phi-\phi_{0}\right)}{c}}, e^{\frac{j 2 \pi f r \cos \left(\phi-\phi_{1}\right)}{c}}, \cdots, e^{\frac{j 2 \pi f \cos \left(\phi-\phi_{K-1}\right)}{c}}\right]^{T}
$$

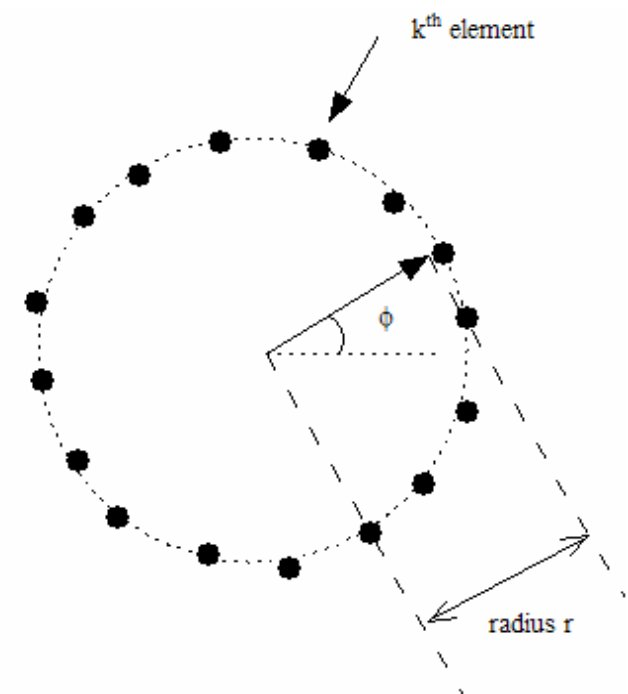

Fig. 1. Uniform Circular Array Configuration

For notation convenience, let $\omega$ denote the digital frequency, i.e. $\omega=\frac{2 \pi f}{f_{s}}, \varepsilon$ denote the ratio of the sampling frequency to the maximum frequency, i.e. $\varepsilon=f_{s} / f_{\max }$, and $\tilde{r}$ denotes the normalized radius, i.e. $\tilde{r}=r / \lambda_{\min }$, the steering vector can be represented as

$\mathbf{a}(\omega, \phi)=\left[e^{j \omega \tilde{r} \varepsilon \cos \left(\phi-\phi_{0}\right)}, e^{j \omega \tilde{r} \varepsilon \cos \left(\phi-\phi_{1}\right)}, \cdots, e^{j \omega \tilde{r} \varepsilon \cos \left(\phi-\phi_{K-1}\right)}\right]^{T}$

Fig 2 shows the system structure of the proposed uniform circular array beamformer. Assuming the sampled signal after the sensor is given by the vector $X[n]=\left[x_{0}(n), x_{1}(n) \cdots, x_{k-1}(n)\right]$, where $\mathrm{n}$ is the sampling instance and $\mathrm{k}$ is the number of sensor, this signal is first transformed into phase mode via Inverse Digital Fourier Transform (IDFT). A compensation filter network which is characterized by the filter coefficient $b_{m}[n]$ is then designed to remove the frequency dependency of the received signal $\mathrm{X}[\mathrm{n}] . h_{m}$ is the spatial weighting coefficient which governs the far field beampattern of the beamformer. $\mathrm{L}$ is defined as $(\mathrm{M}-1) / 2$, where $\mathrm{M}$ is the number of phase mode and it is assumed to be an odd number. The detailed derivation of the spatial response of the beamformer can be found in [1]. In this paper, the proposed beamformer consists of single layer, hence the spatial response of the beamformer is:

$G(\omega, \phi)=\sum_{m=-L}^{L} h_{m} \cdot e^{j m \phi} \cdot K \cdot j^{m} J_{m}(\omega \tilde{r}) \cdot B_{m}(\omega)$

Where $\mathrm{h}$ is the spatial weighting, and $b_{m}[n]$ is the coefficients of the frequency compensation filter and $J_{m}(\beta)$ is the Bessel function of the first kind of order m. $B_{m}(\omega)$ and $b_{m}[n]$ are a Fourier Transform pair which satisfied the 
following relationship: $B_{m}(\omega)=\sum_{n=0}^{N n} b_{m}[n] e^{-j n \omega}$

(2), where

$\mathrm{Nn}$ is the order of the compensation filter.

\section{PROPOSED NOVEL BEAMFORMER}

In order to achieve our goal, the following objective function is formulated for the proposed beamformer:

$$
\min \quad v=\iint_{\omega \phi}\|G(\omega, \phi)\|^{2} d \omega d \phi
$$

Subject to $\quad\left\|G\left(\omega, \phi_{0}\right)-1\right\| \leq \delta, \omega \in\left[\omega_{l}, \omega_{u}\right]$

Where $G(\omega, \phi)$ is the spatial response of the beamformer as shown in equation (1), $\omega_{l}$ and $\omega_{u}$ are the lower and upper limit of the desired frequency range. $\phi_{0}$ is the desired direction.

In this objective function, the square of the overall beampattern across all frequency and all angles is minimized, while the beampattern pertaining to the specific angle is constrained to be approximately one. The main novelty is that it only restricts the FI character of the beampattern along the desired direction for a range of frequency band instead of across all directions. It is done by optimizing the coefficient of the compensation filter and the beam weight together. Cholesky factorization is later used to transform further the objective function into SeDuMi solvable min-max optimization problem. Simulation shows that as the angle deviates from the desired direction, the FI character becomes less obvious for different frequencies. The physical interpretation of the above objective function for being able to achieve FI in single step is that by focusing the FI characteristic at the desired direction for a range of frequency band only and relaxing the FI at other directions, more degrees of freedom are freed. Comparing to UCCA, it has strict FI characteristic at all directions for a wide selection of frequency range. Hence the free degrees of freedom make the proposed beamformer possible to achieve FI in single step. In the next section, the numerical results also points out that with the same array configuration, the proposed beamformer can also yield better performance on achieving frequency invariant beampattern at the desired direction for a wide range of frequency.

In this optimization problem, Second Order Cone Programming (SOCP) is used to solve the above objective function due to the quadratic constraint [12]. SOCP is a convex programming problem and the global optimal solution is guaranteed if it exists. A standard form of SOCP can be written as follows:

$$
\begin{aligned}
& \min \mathbf{b}^{T} \mathbf{x} \\
& \text { Subject to } \mathbf{d}_{i}^{T} \mathbf{x}+q_{i} \geq\left\|\mathbf{A}_{i} \mathbf{x}+\mathbf{c}_{i}\right\|_{2}, i=1, \ldots, N
\end{aligned}
$$

Where $\mathbf{x} \in R^{m}$ is the variable vector; $\mathbf{b}, \mathbf{d}_{i} \in R^{m}, \mathbf{c}_{i} \in R^{m}$, and $q_{i} \in R^{n_{i} \times m}$ are constant vectors; $\mathbf{A}_{i} \in R^{n_{i} \times m}$ are constant

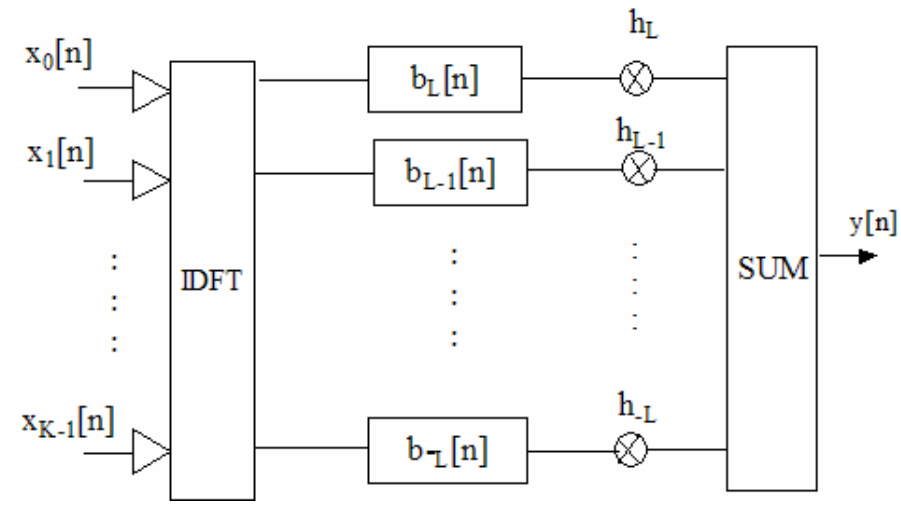

Fig. 2. The system structure of a uniform circular array beamformer

matrices; and $\|u\|_{2}$ denotes the Euclidean norm of the vector $\mathrm{u}$, $\|u\|_{2}=\left(u^{T} u\right)^{\frac{1}{2}}$.

To convert to the standard form, the objective function has to be suitably modified. To start with, substitute (2) into (1),

$$
G(\omega, \phi)=\sum_{m=-L}^{L} h_{m} \cdot e^{j m \phi} \cdot K \cdot j^{m} J_{m}(\omega \tilde{r})\left[\sum_{n=0}^{N n} b_{m}[n] e^{-j n \omega}\right]
$$

Using the identity $e^{-j n \omega}=\cos (n \omega)-j \sin (n \omega)$, equation (5) becomes:

$$
\begin{aligned}
G(\omega, \phi)= & \sum_{m=-L}^{L} h_{m} \cdot e^{j m \phi} \cdot K \cdot j^{m} J_{m}(\omega \tilde{r}) \\
& \cdot\left[\sum_{n=0}^{N n} b_{m}[n](\cos (n \omega)-j \sin (n \omega))\right] \\
= & K \sum_{m=-L}^{L} h_{m} \cdot e^{j m \phi} \cdot j^{m} J_{m}(\omega \tilde{r}) \\
& \cdot\left[\sum_{n=0}^{N n} b_{m}[n] \cos (n \omega)-j b_{m}[n] \sin (n \omega)\right] \\
= & K \sum_{m=-L}^{L} h_{m} \cdot e^{j m \phi} \cdot j^{m} J_{m}(\omega \tilde{r}) \\
& \cdot\left[\sum_{n=0}^{N n} b_{m}[n] \cos (n \omega)-j \sum_{n=0}^{N n} b_{m}[n] \sin (n \omega)\right] \\
= & K \sum_{m=-L}^{L} h_{m} \cdot e^{j m \phi} \cdot j^{m} J_{m}(\omega \tilde{r}) \\
& \cdot\left[\mathbf{c}_{m} \overline{\mathbf{b}} m-j \mathbf{s}_{m} \overline{\mathbf{b}}_{m}\right]
\end{aligned}
$$

where

$$
\begin{aligned}
& \overline{\mathbf{b}}_{m}=\left[b_{m}[0], b_{m}[1], \cdots, b_{m}[N n]\right]^{T} \\
& \mathbf{c}_{m}=[\cos (0), \cos (\omega), \cdots, \cos (N n \cdot \omega)] \\
& \mathbf{s}_{m}=[\sin (0), \sin (\omega), \cdots, \sin (N n \cdot \omega)] \\
& G(\omega, \phi)=K \sum_{m=-L}^{L} h_{m} \cdot e^{j m \phi} \cdot j^{m} J_{m}(\omega \tilde{r}) \mathbf{c}_{m} \overline{\mathbf{b}} m \\
& \quad-j \cdot K \sum_{m=-L}^{L} h_{m} \cdot e^{j m \phi} \cdot j^{m} J_{m}(\omega \tilde{r}) \cdot \mathbf{s}_{m} \overline{\mathbf{b}}_{m}
\end{aligned}
$$


Let $x_{m}=h_{m} \cdot j^{m} \cdot \overline{\mathbf{b}}_{m}$

$$
\begin{aligned}
G(\omega, \phi) & =K \sum_{m=-L}^{L} e^{j m \phi} J_{m}(\omega \tilde{r}) \mathbf{c}_{m} x_{m}-j \cdot K \sum_{m=-L}^{L} e^{j m \phi} J_{m}(\omega \tilde{r}) \cdot \mathbf{s}_{m} x_{m} \\
& =\overline{\mathbf{C}} \mathbf{X}-j \overline{\mathbf{S}} \mathbf{X}
\end{aligned}
$$

where

$$
\begin{aligned}
& \overline{\mathbf{C}}(\omega, \phi)=\left[K e^{j(-L) \phi} J_{-L}(\omega \tilde{r}) \mathbf{c}_{-L}, \cdots, K e^{j(L) \phi} J_{L}(\omega \tilde{r}) \mathbf{c}_{L}\right] \\
& \mathbf{X}=\left[x_{-L}, x_{-L+1}, \cdots, x_{L}\right]^{T} \\
& \overline{\mathbf{S}}(\omega, \phi)=\left[K e^{j(-L) \phi} J_{-L}(\omega \tilde{r}) \mathbf{s}_{-L}, \cdots, K e^{j(L) \phi} J_{L}(\omega \tilde{r}) \mathbf{s}_{L}\right]
\end{aligned}
$$

By stacking $\overline{\mathbf{C}}(\omega, \phi)$ and $\overline{\mathbf{S}}(\omega, \phi)$ together, the above equation can be rewritten in the following form:

$$
G(\omega, \phi)=\left(\begin{array}{l}
\overline{\mathbf{C}}(\omega, \phi) \\
-j \overline{\mathbf{S}}(\omega, \phi)
\end{array}\right) \mathbf{X}=\mathbf{A}^{H} \mathbf{X}
$$

Hence,

$$
\|G(\omega, \phi)\|^{2}=G^{H} G=\left(\mathbf{A}^{H} \mathbf{X}\right)^{H}\left(\mathbf{A}^{H} \mathbf{X}\right)=\mathbf{X}^{H} \mathbf{A}(\omega, \phi) \mathbf{A}(\omega, \phi)^{H} \mathbf{X}
$$

since the objective function is $v=\iint_{\omega}\|G(\omega, \phi)\|^{2} d \omega d \phi$, and $\mathrm{X}$ is independent of all the frequency and angles,

$$
\begin{aligned}
v & =\int_{\omega} \int_{\phi}\left(\mathbf{X}^{H} \mathbf{A}(\omega, \phi) \mathbf{A}(\omega, \phi)^{H} \mathbf{X}\right) d \omega d \phi \\
& =\mathbf{X}^{H}\left(\iint_{\omega} \mathbf{A}(\omega, \phi) \mathbf{A}(\omega, \phi)^{H} d \omega d \phi\right) \mathbf{X} \\
& =\mathbf{X}^{H} \mathbf{M} \mathbf{X}
\end{aligned}
$$

where $\mathbf{M}=\iint_{\omega \phi} \mathbf{A}(\omega, \phi) \mathbf{A}(\omega, \phi)^{H} d \omega d \phi$

Hence, the objective function can finally be written as:

$$
\begin{gathered}
\min \mathbf{X}^{H} \mathbf{M X} \\
\text { Subject to }\left\|\mathbf{A}^{H} \mathbf{X}-\left(\begin{array}{l}
1 \\
0
\end{array}\right)\right\| \leq \delta \text { for } \omega \in\left[\omega_{l}, \omega_{u}\right]
\end{gathered}
$$

Since in the standard SeDuMi form, the objective function must be a linear equation, hence to transform it into (4), Cholesky factorization is used. By definition, Cholesky factorization decomposes symmetric positive definite matrix into transpose of upper triangular matrix and the upper triangular matrix. $M=U^{H} U$, if $\mathrm{M}$ is hermitian and positive definite. Substitute the above relationship into (6), $\mathbf{X}^{H} \mathbf{M X}=\mathbf{X}^{H}\left(U^{H} U\right) \mathbf{X}=(U \mathbf{X})^{H}(U \mathbf{X})$, where $U=\operatorname{chol}(\mathbf{M})$ is the cholesky factorization in Matlab function. Defining $t$ as the maximum value of $U \mathbf{X}$, min-max criterion is skillfully applied so as to convert the minimization of a quadratic term into minimization of a linear term.
Finally, (6) can be written in a matrix form as follows:

$$
\begin{gathered}
\text { min } t \\
\text { Subject to }\|\mathbf{c h o l}(M) \cdot \mathbf{X}\| \leq t \\
\left\|\mathbf{A}\left(\omega, \phi_{0}\right)^{H} \mathbf{X}-\left(\begin{array}{l}
1 \\
0
\end{array}\right)\right\| \leq \delta \quad \text { for } \omega \in\left[\omega_{l}, \omega_{u}\right]
\end{gathered}
$$

By dividing $\omega$ uniformly within the frequency range with a sufficient number of points, says $\mathrm{P}=64$, each continuous constraint above for each angle will yield $\mathrm{P}$ different constraints. The resulting problem is now in the form of a standard SOCP problem, which can be solved efficiently using optimization toolbox such as SeDuMi [11].

\section{NUMERICAL RESULTS}

Consider a single layer of circular array of 20 elements that are arranged as shown in Fig 1. The number of phase mode $\mathrm{M}$ is 17 . Therefore we have 17 spatial weighting coefficients. We assume that the orders of the compensation filter are the same for all phases which is 16 in this experiment. The desired angle of direction of arrival is set to be at broadside. The range of frequency which achieves frequency invariant is from $0.3 \pi$ to $0.95 \pi$. $\delta$ is set to be 0.1 . Due to the reason that the proposed beamformer adapts the same structure as UCCA, in this section, the performance of the two beamformers for the same array setting are compared.

The simulated array beampattern for the proposed beamformer is shown in Fig 3. By dividing the frequency range from $0.3 \pi$ to $0.95 \pi$ uniformly with 10 points, each frequency point corresponds to one blue curve. In Fig 3, it is shown clearly that at broadside, there is little frequency variance in the spatial response. The proposed beamformer is able to achieve frequency invariant at the desired direction. As the angle deviates from broadside direction, the frequency invariant characteristic relaxes for different frequencies. As shown in the figure, the blue curves are no longer overlapping. The sidelobe level is approximately $-10 \mathrm{~dB}$.

In Fig 4, the normalized spatial response of UCCA

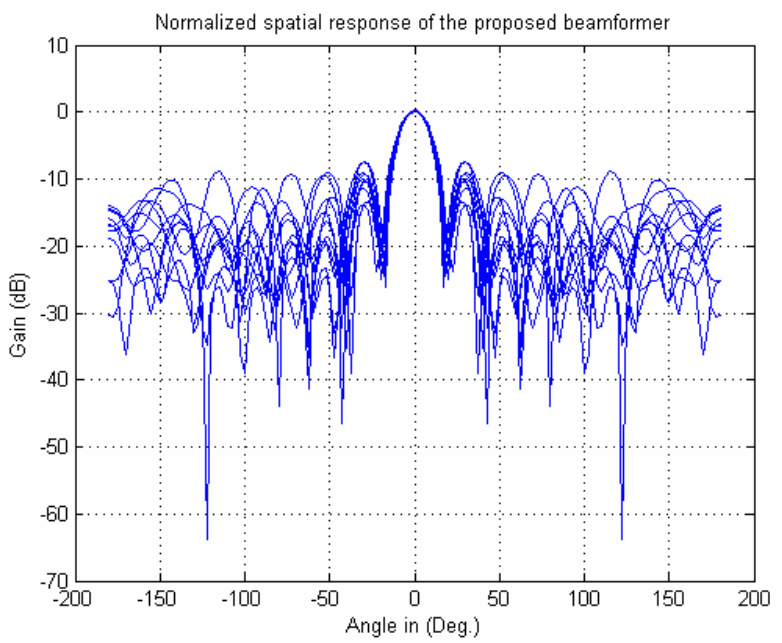

Fig.3. The normalized spatial response of the proposed beamformer for $\omega=[0.3 \pi, 0.95 \pi]$ 
beamformer is presented. In this experiment, instead of using concentric array, single layer of circular array is used. By dividing the frequency range from $0.3 \pi$ to $0.95 \pi$ uniformly with 10 points again, each frequency point corresponds to one red curve. From the beampattern, we could see that for UCCA beamformer, at the broadside, there is some degree of frequency variance. It is not as smooth as the proposed beamformer. As the angle gets further from the desired direction, the beampattern differ more for different frequencies. The sidelobe level is approximately $-15 \mathrm{~dB}$. From here, we could see that for the same frequency range as stated in [1], the proposed beamformer achieves better frequency-invariant characteristic than UCCA beamformer for the same circular array setting.

In Fig 5, comparison between the proposed beamformer and the UCCA beamformer on FI characteristic across all frequency is presented. The blue line represents the proposed beamformer, while the red line represents the UCCA beamformer. It clearly shows that at the desired direction, the proposed beamformer obtain better performance on FI characteristic than UCCA beamformer.

When the frequency range is modified from $[0.3 \pi, 0.95 \pi]$ to $[0.2 \pi, 0.3 \pi]$, the proposed beamformer achieves FI at the desired direction with narrower main beam but higher sidelobe as compared to UCCA beamformer.

\section{CONCLUSIONS}

In this paper, a novel beamformer that achieves frequencyinvariant characteristic for uniform circular array in single step is proposed. Simulations results show that the proposed beamformer performs well for both short range of frequency and wide range of frequency. More importantly, the optimization is done in single step. This is useful in many real-time applications, especially for speech capturing in vehicular environment.

\section{ACKNOWLEDGMENT}

The authors would like to acknowledge the helpful discussions concerning the simulation of UCCA with $\mathrm{H}$. H. Chen of The University of Hong Kong. They would also like to thank ST Micro for the sponsorship of this project.

\section{REFERENCES}

[1] S. C. Chan and H. H. Chen, "Uniform Concentric Circular Arrays With Frequency-Invariant Characteristics- Theory, Design, Adaptive Beamforming and DOA Estimation,” IEEE Trans. Signal Processing., vol. 55, no. 1, January 2007

[2] Barry D.Van Veen and Kevin M. Buckley, "Beamforming: A versatile approach to spatial filtering," IEEE, Trans. Acoust, Speech, Signal Processing, April 1988

[3] Hamid Krim and Mats Viberg, "Two decades of Array signal Processing Research,” IEEE Signal Processing Magazine, July, 1996

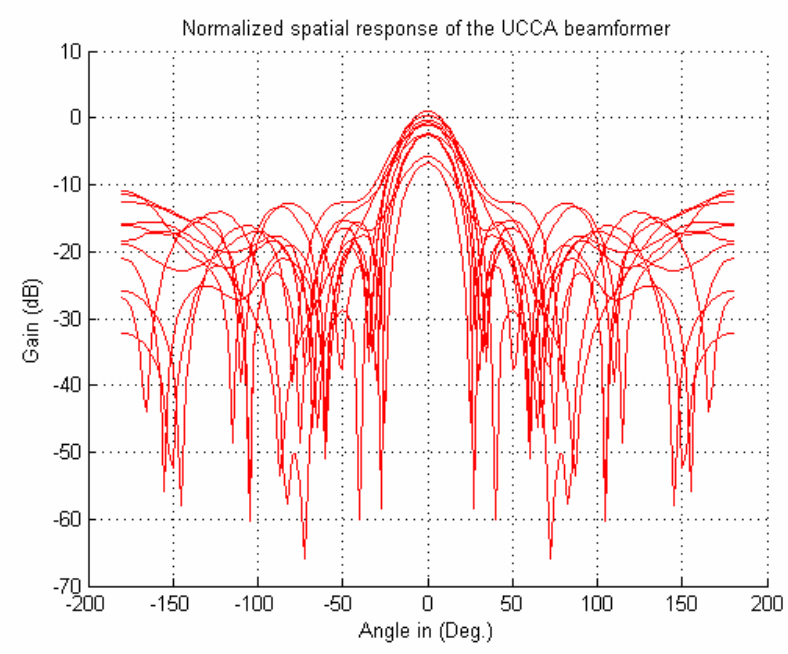

Fig.4. The normalized spatial response of the UCCA beamformer for $\omega=[0.3 \pi, 0.95 \pi]$

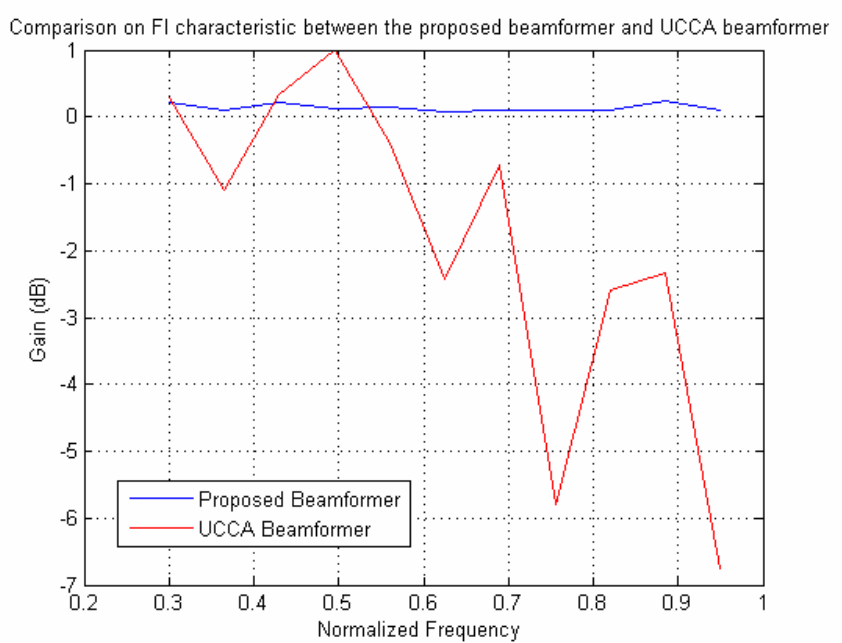

Fig.5. Comparison on FI characteristic between the proposed beamformer and UCCA beamformer across all frequency at 0 degree

[4] O. L. Frost, "An algorithm for linearly constrained adaptive array processing” No. 8. August 1972 in Proceedings of the IEEE. vol. 60,

[5] Don H. Johnson and Dan E. Dudgeon, Array Signal Processing: concepts and Techniques, Upper Saddle River, N.J.: Prentice Hall, 1993

[6] Robert A. Monzingo, and Thomas W. Miller, Introduction to Adaptive Arrays, John Wiley \& Sons Inc, SciTech Publishing, 2004

[7] D.B.Ward, R.A.Kennedy, "FIR filter design for frequency invariant beamformers,” IEEE, Signal Process.Lett.,vol.3,pp.6971, Mar. 1996

[8] Andrea Trucco and Stefania Repetto, "Frequency Invariant Beamforming in Very Short Arrays,” IEEE Conference. 2004

[9] Andrea Trucco, Marco Crocco, and Stefania Repetto, “A Stochastic Approach to the Synthesis of a Robust FrequencyInvariant Filter-and-Sum Beamformer," IEEE Trans. Instrumentation and measurement, vol. 55, no. 4, August. 2006 
[10] D.B.Ward, R.A.Kennedy, "Theory and design of broadband sensor arrays with frequency invariant far-field beam patterns," J.Acoust.Soc.Amer, vol.97, no.2,pp.1023-1034, Feb 1995

[11] J.F.Sturm, "Using SeDuMi 1.02, a MATLAB toolbox for optimization over symmetric cones," Optim. Meth. Softw., vol. 11-12, pp. 625-653, 1999

[12] M.S.Lobo, L.Vandenberghe, S.Boyd, and H. Lebret, "Applications of second-order cone programming," Linear Algebra Appl., vol. 248, pp. 193-228, Nov. 1998

[13] I.D. Dotlic, "Minimax frequency invariant beamforming," IEEE, Electronic letters, vol. 40, No. 19, Sep 2004

[14] M. Ghavami, "Wideband smart antenna theory using rectangular array structures," IEEE transactions on Signal Processing, vol. 50, no. 9, pp. 2143-2151, Sep 2002 